\title{
Fingerprint Recognition based on Minutiae Information
}

\author{
Chandana \\ M.Tech Student, \\ Dept. of CSE, \\ MACERC, Sirsi Jaipur
}

\author{
Surendra Yadav, PhD \\ Professor \& Head, \\ Dept. of CSE, \\ MACERC, Sirsi Jaipur
}

\author{
Manish Mathuria \\ Assistant Professor, \\ Dept. of CSE, \\ MACERC, Sirsi Jaipur
}

\begin{abstract}
The performance of Fingerprint Recognition is totally dependent on the Fingerprint Image Quality, because Fingerprint Recognition is the processing of Digital Image. The quality of fingerprint images influences by both environmental and scanner's surface. This paper analyzes the Fingerprint Recognition, Matching. The Minutiae-based matching technique is the best method to extract useful information about Minutiae and if the image is partial or affected by noise then the ROI can be use to optimize the result. Authentication using fingerprint has been implementing in many areas (like Attendance System, Bank Desktop Verification Login). UID is the recent project of Govt. India focused on Recognition of Identity by using Fingerprint and Retina.
\end{abstract}

The conclusion state about how minutiae points are important to recognize fingerprint and ROI (the specified limited area) to avoid error within less time. Whereas, 'one to one matching' can quickly reply compare to 'one to many matching'. Future scope of "Fingerprint Recognition based on Minutiae" has wide range of application areas such as Mobile Computing, Bank ATM, NAREGA Identity Verification, etc.

\section{INTRODUCTION}

A person's behavioral or physiological characteristics can be identified using the Biometric Techniques. The 'Biometric' is derived from Greek words "bios" means Life and "metric" means Measurement. Human behavioral characteristics (voice, signature etc) can be changed with time. But their physiological characteristics such as fingerprint, retina etc. can never be changed. Biometric process gives the highly secure authorization process between an unauthorized and a genuine person. Biometric Authentication process, first match the information with stored data i.e. Verification and then identify the person i.e. Identification. [1]

The Fingerprint Recognition is not a latest technique for Identification. The fingerprints were found on ancient Babylonian clay tablets, seals, and pottery. The Fingerprints were used as signatures for illiterate person. Those fingerprint fall into Patent Fingerprint category, and recognized by eyes using lenses without computer processing. But now, it is possible to scan patent fingerprint to develop its digital image that can be recognized after processing algorithms. The modern use of computer and mobile devices is becoming more dangerous to leak private information that need to be protected. The password protection is not sufficient to draw authentication scenario for hackers, it require some additional security layers. The Fingerprint Recognition is the best bridge to connect original user to the belonging services. The question arrives "How is much efficient to Fingerprint Authenticate?", "Is it possible to create fake Fingerprint
Image?", "Why fingerprint is still not fully implemented in Industries, Societies, and local areas?", "What are the Govt. Initiatives to Fingerprint based Security and Recognition?", and "How it can be feasible to store and retrieve fingerprints of large group of people?" The solution to these questions is the main focus of the research.

\section{FINGERPRINT RECONIGATION}

Fingerprint recognition is also called as fingerprint authentication. It is a most popular biometric solution, refers to the automated method to confirmation the identity.

- One to One Matching:- It is applied where Input fingerprint is matched directly with only one fingerprint, which produce result either matched or not matched. Example: Secure Login using EmailID, Laptop \& Desktop Device Protection, etc.

- One to Many Matching:- It is applied to specified areas where number of applicant store their fingerprint. The Input fingerprint is matched with number of fingerprint stored to uniquely find person identity. Example: Attendance Management, Secure Login without using EmailID, etc.

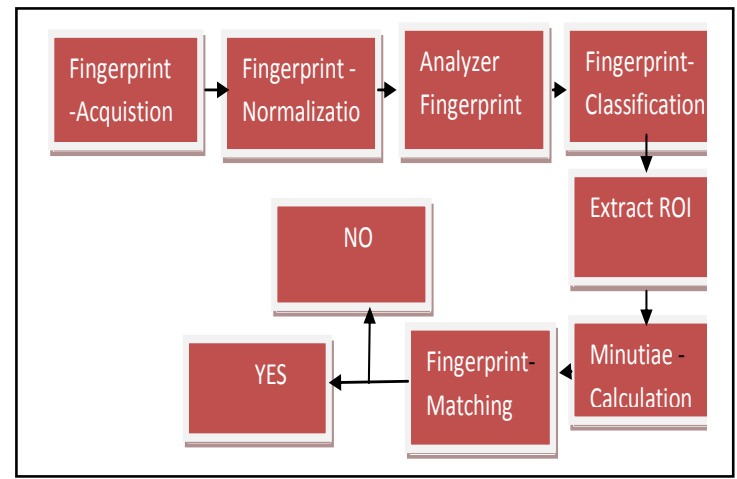

Fig 1: Basic Steps of Fingerprint Recognition System

Due to its cheapest biometric solution fingerprint recognition uses in different areas such as Physical Access Control and Time \& Attendance Management.

\subsection{Importance of Fingerprint:}

Fingerprint is a very interesting and unique feature of the human body, because the Fingerprints can uniquely identify the person and it does not changes from birth to death. Even twines' fingerprints do not match. Due to persistent and uniqueness property of fingerprint it has been implementing in many areas such as UID Card, Passport, Criminal Data, etc.

Fingerprints are unique pattern of ridges (raised) and furrows (recessed). That appears on the pads of fingers and thumbs. Fingerprints Acquisition Techniques are categorized into three types [2]. 
2.1.1 Live Scan Fingerprints: Live Scan Fingerprints are Impressed Fingerprints sometimes known as Plastic Prints. The quality of Fingerprint image received is better than Patent Fingerprint. It can be retrieve using fingerprint scanner, Clay, Wax, and Paint.

2.1.2 Latent Fingerprints: Latent fingerprint is invisible to the eye directly and requires enhancement technique and instruments to check. To extract the fingerprint from the surface basic powder and chemical techniques are used.

2.1.3 Patent Fingerprint: Patent Fingerprint is also called as the Visible Fingerprint. It is formed intentionally by person to proof their identity. At identification stage, it is still use to record presence of person. Patent Fingerprints can be created by blood, grease, ink, or dirt etc.

\section{FINGERPRINT CLASSIFICATION}

The Classification fingerprint images are very important to speed up the recognition process in which fingerprints are classified according their shapes of Minutiae Ridges. Populations around the world have different types of fingerprint patterns those are as follows:

3.1 Arch Pattern: The Arch is a type of ridges. which start from a side and ending to opposite side by making wave pattern at centre. There are two type of Arch Pattern named Plain Arch and Tent Arch[3]

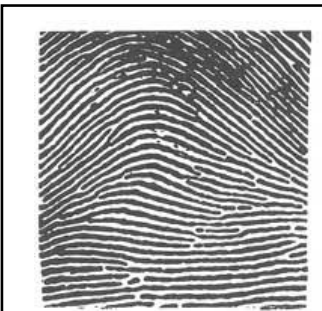

(a) Plain Arch

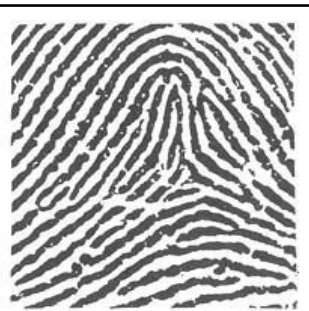

(b) Tented Arch
Fig 2: Loop Pattern

3.2 Loop Pattern: Loop is the special property of fingerprint image. There are many types of loops are available such as Right Loop, Left Loop, Double Loop, Left Pocket Loop, Right Pocket Loop Pattern. Mostly, Right and Left loops are found in fingerprint of human. The loop is towards the right or Ulna bone known as "Ulnar loop".

The loop is towards the left or Radial bone is known as "Radial Loop".

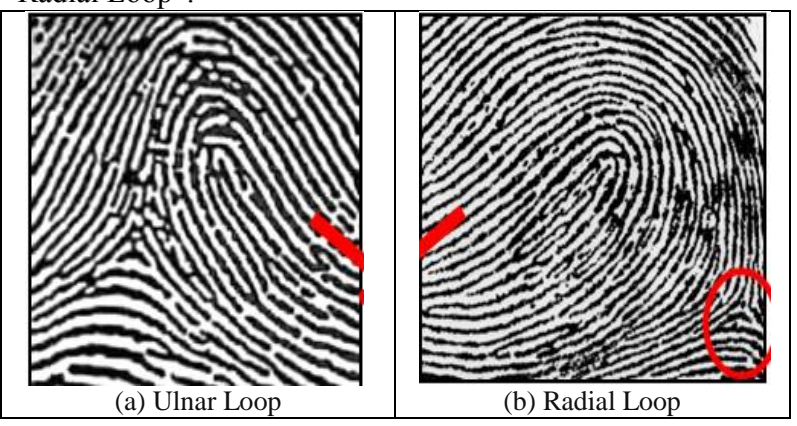

Fig 3: Loop Pattern
3.3 Whorl Pattern: Whorl Pattern is one of the basic patterns of the human fingerprint that have at least one ridge that tends to make a complete circuit. Types of Whorl attern are Plain Whorl, Double Loop Whorl, Central Pocket Loop Whorl, and Accidental Whorl.

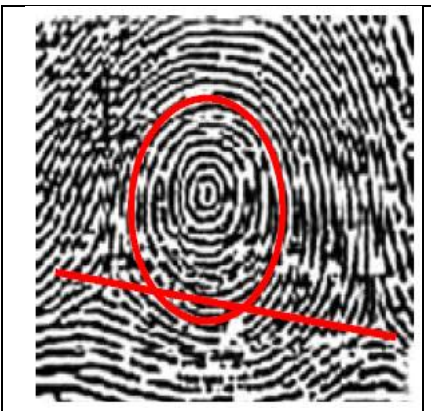

(a) Plain Whorl

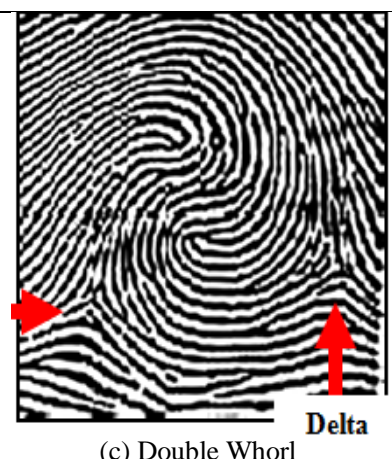

(c) Double Whorl

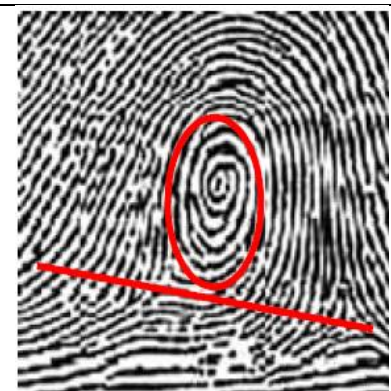

(b) Central Pocket Whorl

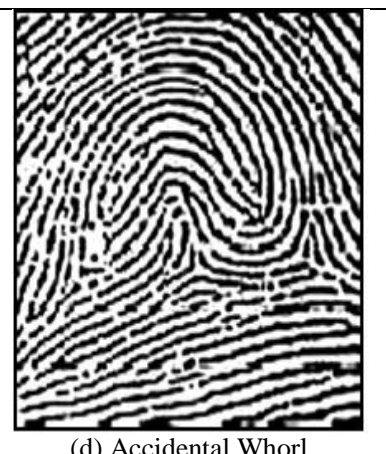

Fig 4: Whorl Pattern

The results of survey to judge which fingerprint pattern has maximum availability in human population are as follows: [4]

Table 1. Fingerprint Pattern

\begin{tabular}{|c|c|c|}
\hline Sr. No. & Pattern & Percentage \\
\hline 1. & Loop & 65 \\
\hline 2. & Whorl & 30 \\
\hline 3. & Arch Pattern & 5 \\
\hline
\end{tabular}

\section{PARTIAL AND FULL FINGERPRINT}

A person fingerprint Impression or mark made on any surface, able to be used for the unique natural pattern of common matching methods or structure such as delta and core on the tips of the finger. The pattern left on surface or uncovered fingerprints. Where all Minutiae cannot be covered such pattern is called as partial fingerprints. Partial fingerprints can also define as the subset of full fingerprints. The partial fingerprint having a small area as compares to full fingerprint from which to takes measurements of Minutiae. Due to this limiting factor of partial fingerprints it declines the degree of certainty of identity as well as the uniqueness of fingerprints.

4.1 ROI: - Region of interest is a segmentation technique. The main motive of segment is to detect the Reference point (core point) in the fingerprint image and crop a small region centered in the 
Reference point from given input is called as ROI [Region of Interest]. ROI is defined in according to required image resolution window of square shape. It has the sufficient information to represent the fingerprint.

4.2 Orientation Estimation: The meaning of fingerprint Orientation is the action of orienting image to the points of a compass or other specified positions. The angle of orientation field represent the direction of one is facing towards. To obtain the ridge orientation the most popular approach is Gradient Based approach. There are some other approach also proposed for orientation fingerprint such as Filter-bank [5], Spectrum estimation [6] and Waveform projection [7] etc.

\section{FINGERPRINT MATCHING TECHNIQUES}

Fingerprint Matching is a technique which is used to match Fingerprint Impression of human with others available in database. This technique is a core part of Fingerprint Recognition process which includes individual analysis of fingerprint and image properties. The result of Fingerprint Matching is Matched Score and Percentage of similarity. The next stage after Fingerprint Matching is Decision Making process based on previous experience.

\subsection{Type of Matching Techniques:}

5.1.1 Minutiae-based Matching: Minutiae is special image property in Fingerprint image which make it possible to classify fingerprint patterns as well as matching based on Minutiae structure and ending (points). When there was no digital technology to get digital fingerprint scan image that time the fingerprints are recognized by person (Fingerprint Analyzer) viewing directly physical impression using lens for zooming. Actually, the Analyzer analyzes the Minutiae appearance and took record of Minutiae Pattern and total count of Minutiae Lines and Points. That is why Minutiae-Based Matching Technique is applicable on all types of digital fingerprint images i.e. Latent, Patent, and Live Scan. The Ridges and Bifurcation points are the very important factor to Minutiae-based matching, to find and recognize Ridges and Bifurcation point is the first step of post processing of fingerprint image in Minutiae matching. In Fingerprints minutiae information approach, Minutiae points (i.e. Ridges and Bifurcation points) are calculates with their locations as well as comparison is performed with the stored fingerprint's Minutiae points.

\subsubsection{Correlation Based Matching: It is advanced} Matching Technique which involves advance image processing methods. The Correlation-based matching processes to correlate pixel values of fingerprint image instead of Minutiae. The specific color properties and threshold values are store to correlate with template stored in the database. It is very helpful when Minutiae information from fingerprint image is not able to extract. [8]

5.1.3 Ridge Feature Based Matching: It is an advanced version of Minutiae based matching. First step of the matching is to find available ridges in the fingerprint image. Then select some ridges to process for extraction. Primarily, extracted ridges passed to normalized phase and classified according to their structure using store information. These ridges then match according to their class pattern and location information.

\subsection{Classification of Matching Parameter:}

The Classification fingerprint images are very important to speed up the recognition process in which fingerprints are classified according their shapes of Minutiae Ridges. So that partial or full fingerprints of same finger can easily be match to same group. If the Database of fingerprint images contains several million Digital Fingerprint Impressions, then it will take a long response time. Hence, to overcome this problem the fingerprint classification method is used. The fingerprint matching classification can be categorized as follows [9].

TABle 2. Fingerprint MATChingClassification

\begin{tabular}{|c|l|}
\hline Patterns & $\begin{array}{l}\text { The flow pattern of Minutiae in } \\
\text { a fingerprint is unique to the } \\
\text { person. }\end{array}$ \\
\hline Minutiae points & $\begin{array}{l}\text { It refers to the Minutiae ending } \\
\text { known as Ridges and } \\
\text { Bifurcation points. }\end{array}$ \\
\hline Ridge contours & $\begin{array}{l}\text { The shape and structure of } \\
\text { Ridge. }\end{array}$ \\
\hline Location: & $\begin{array}{l}\text { Locate the coordinate value for } \\
\text { each ridges \& bifurcation point, } \\
\text { ridges pores. }\end{array}$ \\
\hline Ridge Pore: & $\begin{array}{l}\text { Calculating numbers of ridge } \\
\text { pore in ROI and store location. }\end{array}$ \\
\hline
\end{tabular}

\subsection{Role of Minutiae Point}

A minutia is defined as points of interest in a fingerprint such as Termination [Ridge ending] and Bifurcation [Ridge splits into two parts] points.

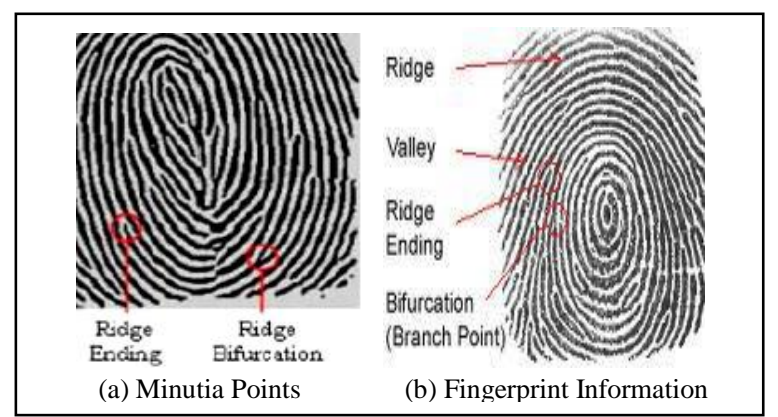

Fig 5: Basic properties of Fingerprint Image. [10,11]

A fingerprint is made by many ridge and valleys. The continuous Black pattern flow is called as "Ridge" and the White pattern between the ridges is called as "Valley". [12]

Table 3. Fingerprint Shape Properties

\begin{tabular}{|c|l|}
\hline Ridges End & The peek end of a ridges \\
\hline Bifurcation End & Ridge decomposes into two ridges. \\
\hline $\begin{array}{c}\text { Independent } \\
\text { Ridge }\end{array}$ & It is a short length ridges. \\
\hline Island & $\begin{array}{l}\text { A ridge ending which is not } \\
\text { connected to any other ridge. }\end{array}$ \\
\hline
\end{tabular}




\begin{tabular}{|c|l|}
\hline Spur & $\begin{array}{l}\text { a bifurcation with a short ridge } \\
\text { branching off a longer ridge }\end{array}$ \\
\hline $\begin{array}{c}\text { Crossover or } \\
\text { Bridge }\end{array}$ & $\begin{array}{l}\text { A short ridge that runs between two } \\
\text { parallel ridges. }\end{array}$ \\
\hline Delta & $\begin{array}{l}\text { Delta is define as a Y- shaped ridge } \\
\text { pattern }\end{array}$ \\
\hline Core & A U- turn in the ridge pattern \\
\hline
\end{tabular}

\section{RECENT DEVELOPMENT}

Recently, Fingerprint Recognition is using as a key identity method to uniquely distinguish the person from others as well as provides its original details. The areas where the fingerprint is using as a key feature are as follows:

UID (Unique Identification) Card: It's a very large project of Govt. of India. This UID card contains lots of personal information including Fingerprint of 10 Fingers. These images are stored at centralized system which can be accessed from any place of India to authenticate the person (in Banks, Govt. office, Student Exams, Bus-Railways Ticket Reservation, Entry System, Address Verification, and many more applications).

Secure Fingerprint-Based Login System: The Fingerprint Authentication at client machine is used to identify its original user. Only the owner of system can used that device. This system has been implementing in Banks and Laptop Devices.

\section{CONCLUSION}

The conclusion of the research is, the Fingerprint Recognition based on Minutiae Information is the key to solve many problems related to fake entries and security attacks. The fingerprint is the only Biometric type which is very easy to take, handle, and operated using recent developments in Image Processing. The problems regarding image size in database is not a serious problem due to cloud computing whereas unlimited data can be stored and transfer moreover many researches are in process. The important points concluded are as follows:

- Fingerprint Images are very easy to recognize compared to retina images because it has specific pattern to classify.

- In case two Fingerprints of different person are matched, then Fingerprints of other fingers can never be match with other person's fingerprints.

- If the matching techniques discussed above may compose into a single system then the fingerprint verification results will optimized.

\section{FUTURE SCOPE}

Application those provides solutions, support, feedback and problem conclusion. These all are required to be uniquely identifying in future fingerprint can be a most important factor for authentication and authorization. The used of UID to test fingerprint at different places with different application can make it feasible examine the originality of person presented.
$>$ If the Govt. Election may conduct using UID card, then fake entries can be avoided.

$>$ If the ATM Machine and Card may connect with the UID card system then only allowed people would transact money Authenticate by Fingerprint Scanner at ATM.

$>$ Other Scheme those can take advantage to Fingerprint Scanned Images by UID are as follow: Indian Post Office, NREGA.

\section{REFERENCES}

[1] Manvjeet Kaur, Mukhwder Singh, Akshay Girdhar, and Parvindar S. Sandhu "Fingerprint Verification System using Minutiae Extraction Technique" World Academy of Science, Engineering and Technology 22, 2008.

[2] Dr. Neeraj Bhargava, Dr. Ritu Bhargava, Prafull Naroka, Minaxi Cotia, "Fingerprint Recognition Using Minutiae Matching" International Journal of Computer Trends and Technology- Volume 3, 2012.

[3] Federal Bureau of Investigation John Edgar Hoove, The Science of Fingerprints Classification and Uses August 10, 2006.

[4] Wikipedia“Fingerprints", http://en.wikipedia.org/wiki/Fingerprint

[5] A.K. Jain, S. Pankanti , L. Hong, "A multichannel approach to fingerprint classification" IEEE Trans. Pattern Anal. Machine Intell. 21, 348-359, 1999.

[6] C.L. Wilson, G.T. Candela, C.I. Watson, Neural network fingerprint classification, J. Artif. Neural Networks 1, 203-228, 1994.

[7] B.G. Sherlock, D.M. Monro, K. Millard, "Fingerprint enhancement by directional Fourier filtering" IEE Proc. Vision, Image Signal Process. 141, 87-94, 1994.

[8] Asker M. Bazen, Gerben T.B. Verwaaijen, Sabih H. Gerez,Leo P.J. Veelenturf and Berend Jan van der Zwaag, "A Correlation-Based Fingerprint Verification System"University of Twente, Department of Electrical Engineering.

[9] Manish Mathuria, Minaxi Cotia" Fingerprint Classification based on Orientaion Estimation" International Journal of Advanced Research in Computer Science, Volume 4, No. 7, June 2013

[10] Avani Patel, Prof. Vikarm Agarwal, Prof. Vastal H. shah, Improve Fingerprint Recognition Using both Minutiae Based and Pattern Based Method, ISSN:0974-6471

[11] Om Preeti Chaurasi."Amity School of Engineering and Technology, Amity University, Noida, India" An Approach to Fingerprint Image Pre-Processing, Published Online July 2012 in MECS (http://www.mecspress.org/)

[12] Dr. Neeraj Bhargava, Manish Mathuria, Dr. Ritu Bhargava, Minaxi Cotia, "Scope analysis of different kinds of fingerprint" Binary Journal of Data Mining and Networking 1(2010), 15-18, 2013. 